\title{
Applying the new STEMI guidelines: 2. Disturbances of cardiac rhythm after ST-segment elevation myocardial infarction
}

ß See related article page 1039

\begin{abstract}
The authors constitute the Canadian Cardiovascular Society Working Group, formed to review the new American College of Cardiology / American Heart Association STEMI guidelines and to adapt them for Canadian practice. In this second of 2 articles, they describe cases illustrating the care of patients with arrhythmias after STEMI.
\end{abstract}

\begin{abstract}
Case 1
A 52-year-old man with hypertension and hyperlipidemia experiences chest pain and summons medical help. Thirty minutes later, in the presence of paramedics, he collapses with ventricular fibrillation (VF). He is promptly defibrillated. His admission electrocardiogram (ECG) reveals anterior ST-segment elevation. He receives intravenous fibrinolysis.
\end{abstract}

Question: Is this patient likely to have further episodes of VF?

Comment: Whereas VF in the first hour after myocardial infarction (MI) is relatively common, the risk of subsequent $\mathrm{VF}$ is similar to that in patients with an MI of equivalent size but without early VF. ${ }^{1}$ No specific antiarrhythmic therapy is needed for such patients. ${ }^{2}$

Question: Later that day the patient experiences recurrent chest discomfort along with new ECG changes indicating ischemia. Angiography reveals a $90 \%$ proximal lesion in the left anterior descending coronary artery, which is treated with angioplasty and stenting. The angiogram reveals only mild disease in the patient's other $\stackrel{\infty}{\neq}$ coronary arteries. Apart from transient mild heart failure, the patient has no further complications. However, 48 hours after of admission, while reading, he has $\stackrel{-2}{0}$ an episode of presyncope. ECG telemetry shows a 30-second $\curvearrowleft$ episode of polymorphic ven- tricular tachycardia (VT), the first beat having a short coupling interval to the last sinus beat (Fig. 1). The VT stops spontaneously.

What is the clinical significance of this arrhythmia? How should this patient be treated?

Comment: Self-terminating polymorphic VT, particularly in an "ischemic milieu," often occurs during activity or sinus tachycardia, and usually starts with a premature ventricular beat that is "closely coupled" (often for 300 milliseconds or less) to the last normal beat. This is the ECG signature of an ischemia- or infarction-related arrhythmia and is less likely than monomorphic VT to imply a "fixed arrhythmogenic substrate" (myocardial scar tissue from a previous infarct). The appropriate treatment is vigorous anti-ischemic therapy.

Question: Should this patient receive an implantable cardioverter defibrillator (ICD)?
Comment: Patients with this arrhythmia generally do not require an ICD, because the risk of recurrent sustained VT or $\mathrm{VF}$ is much lower than it is after monomorphic VT. ${ }^{3}$

Question: The patient's metoprolol dose is increased from 50 to $100 \mathrm{mg}$ twice daily, and his other medications, including an angiotensin-converting-enzyme inhibitor, ASA, clopidogrel and a statin, are continued. Echocardiography shows moderately severe segmental left ventricular dysfunction, with akinesis in the anterior and inferior septum and apex and an estimated left ventricular ejection fraction of $30 \%$. On day 6, an exercise test with perfusion imaging shows no inducible arrhythmias and no reversible ischemia.

Given the low ejection fraction, is this patient at increased risk of subsequent arrhythmias? Should he receive an ICD before discharge?

Comment: Left ventricular function often improves after revascularization. Decisions regarding the need for ICD insertion should be deferred until at least 40 days after the acute event unless a sustained ventricular arrhythmia occurs beyond the initial 48 hours. If, on reassessment, the left ventricular function is found to be only moderately impaired (ejection fraction $>40 \%$ ), further in-

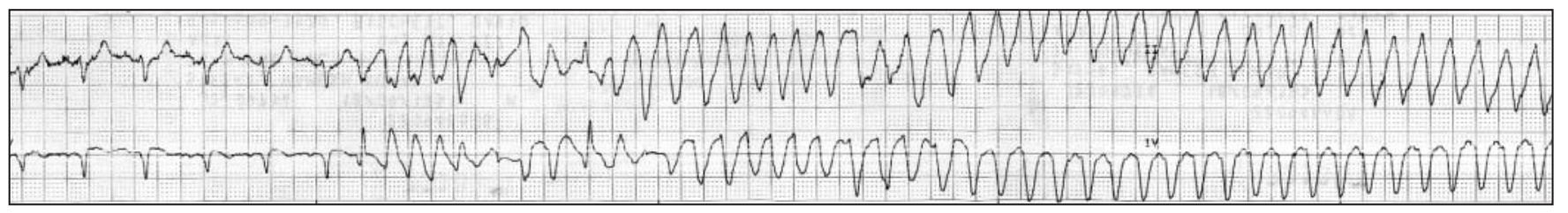

Fig. 1: Polymorphic ventricular tachycardia (VT), arising from sinus tachycardia at 110 beats/min. Note the relatively short QT interval of $\mathbf{2 8 0}$ milliseconds and the short coupling interval from the last sinus beat to the first beat of VT of 300 milliseconds. These features suggest ischemia-related VT. 


\section{Canadian Cardiovascular Society Working Group algorithm}

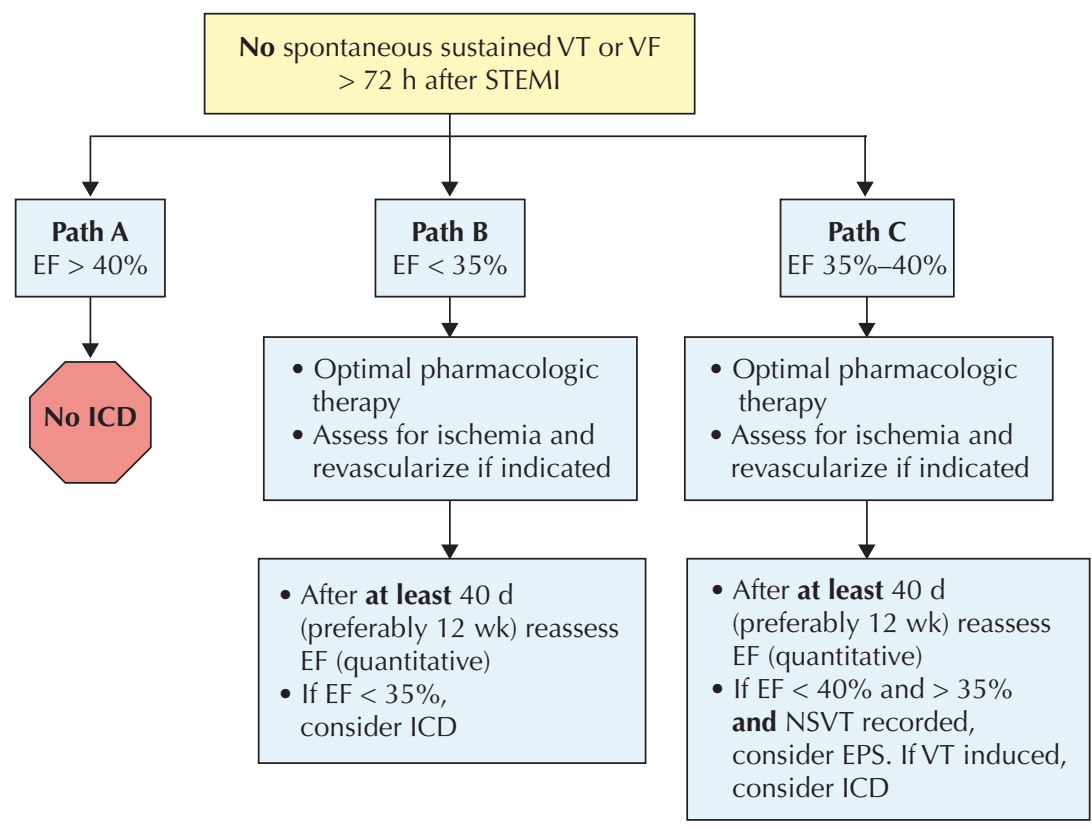

Fig. 2: Canadian Cardiovascular Society Working Group algorithm for the selection of patients for insertion of an implantable cardioverter defibrillator (ICD) after ST-segment elevation myocardial infarction (STEMI). VT = ventricular tachycardia, VF = ventricular fibrillation, EF = ejection fraction, NSVT = nonsustained VT. Reproduced with permission from reference 5.

vestigations with respect to arrhythmia risk, or ICD insertion, are not indicated ${ }^{3,4}$ (Fig. 2).
Outcome: The patient is discharged. Six weeks later, repeat echocardiography reveals mild left ventricular dysfunction (ejection fraction $41 \%)$. He has had no further symptoms of arrhythmias.

\section{Case 2}

A 70-year-old man without prior coronary artery disease has an initially unrecognized MI. Forty-eight hours later he presents with weakness. The ECG shows Q waves and ST-segment elevation consistent with a recent inferior wall $\mathrm{Ml}$; this is confirmed by elevated creatine kinase and cardiac troponin levels. He is treated with ASA, a $\beta$-blocker, an angiotensin-converting-enzyme inhibitor and a statin. Apart from transient heart failure, he has no recurrent ischemia or other early complications. Three days later (5 days after the presumed onset of $\mathrm{MI}$ ), sustained monomorphic ventricular flutter develops at a rate of 280 beats/min, degenerating to ventricular fibrillation (Fig. 3) and requiring immediate defibrillation.

Question: Is this patient at high risk of recurrence of this arrhythmia?

Comment: Sustained monomorphic VT in the presence of a ventricular scar, regardless of how quickly it degenerates to VF, strongly suggests the likeli- hood of recurrent cardiac arrest and requires insertion of an implantable cardioverter defibrillator (ICD). In contrast to polymorphic VT or VF at the outset of arrhythmia, monomorphic VT is a consequence of the ventricular scar from the MI and cannot be prevented by revascularization or antiischemic therapy.

Question: Subsequent cardiac catheterization shows moderate left ventricular dysfunction (ejection fraction $40 \%$ ), inferior akinesis and an occluded right coronary artery with noncritical disease $(<50 \%$ stenosis) of the left anterior descending coronary artery. Should this patient receive an ICD?

Comment: Substantial clinical trial evidence indicates that insertion of an ICD is the best therapy in such cases. Antiarrhythmic therapy, for example with amiodarone, is substantially inferior in both the short and long term for the prevention of sudden cardiac arrest and death., ${ }^{1,3}$ 


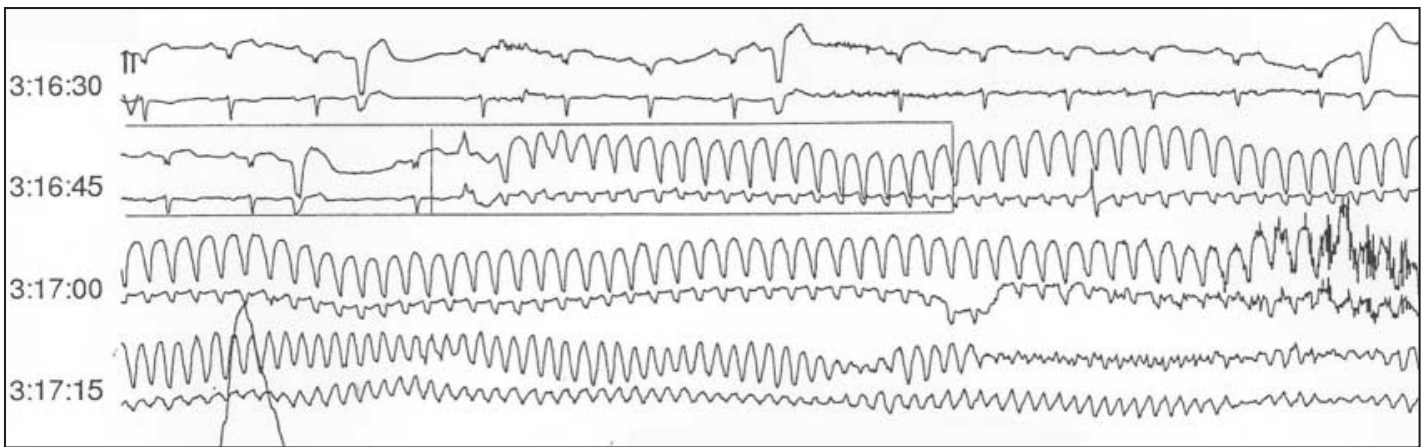

Fig. 3: Sustained, rapid VT quickly degenerating to a polymorphic, disorganized VT and then to VF. The monomorphic pattern at the outset suggests a "fixed arrhythmogenic substrate."

Outcome: The patient underwent implantation of an ICD 3 days later.

\section{Paul Dorian}

Department of Medicine

St. Michael's Hospital

University of Toronto

Toronto, Ont.

Peter Bogaty

Quebec Heart Institute

Laval Hospital

Sainte-Foy, Que.

Christopher E. Buller

Department of Medicine

Division of Cardiology

St. Paul's Hospital

University of British Columbia

Vancouver, BC

Blair J. O'Neill

Department of Medicine

Dalhousie University

Halifax, NS
Paul W. Armstrong

Department of Medicine

University of Alberta

Edmonton, Alta.

Competing interests: Dr. Armstrong has received research funding from HoffmanLaRoche, Aventis, Boehringer Ingelheim, and educational and consultant funding from Hoffmann-LaRoche and Aventis. Dr. Dorian received speaker fees from Guidant Corp., Medtronic Inc., and St. Jude Medical Inc.

\section{References}

1. Bokhari F, Newman D, Greene M, Korley V, Mangat I, Dorian P. Longterm comparison of the implantable cardioverter defibrillator versus amiodarone: eleven-year follow-up of a subset of patients in the Canadian Implantable Defibrillator Study (CIDS). Circulation 2004;110(2):112-6.

2. Antman EM, Anbe DT, Armstrong PW, Bates ER, Green LA, Hand M, et al. ACC/AHA guidelines for the management of patients with ST-elevation myocardial infarction - executive summary: a report of the American College of Cardiology/American Heart Association Task Force on Practice Guidelines (Writing Committee to Revise the 1999 Guidelines for the Management of Patients With Acute Myocardial Infarction). Circulation 2004;110:588-636.

3. Wolpert C, Kuschyk J, Aramin N, Spehl S, Streitner F, Suselbeck T, et al. Incidence and electrophysiological characteristics of spontaneous ventricular tachyarrhythmias in high risk coronary patients and prophylactic implantation of a defibrillator. Heart 2004;90(6):667-71.

4. Connolly SJ, Hallstrom AP, Cappato R, Schron EB, Kuck KH, Zipes DP, et al. Meta-analysis of the implantable cardioverter defibrillator secondary prevention trials. AVID, CASH and CIDS studies. Antiarrhythmics vs Implantable Defibrillator study. Cardiac Arrest Study Hamburg. Canadian Implantable Defibrillator Study. Eur Heart 7 2000;21(24):2071-8.

5. Armstrong PW, Bogaty P, Buller CF Dorian P, O'Neill BJ. The 2004 ACC/AHA guidelines: a perspective and adaptation for Canada by the Canadian Cardiovascular Society Working Group. Can f Cardiol 2004;20(10):1075-9.

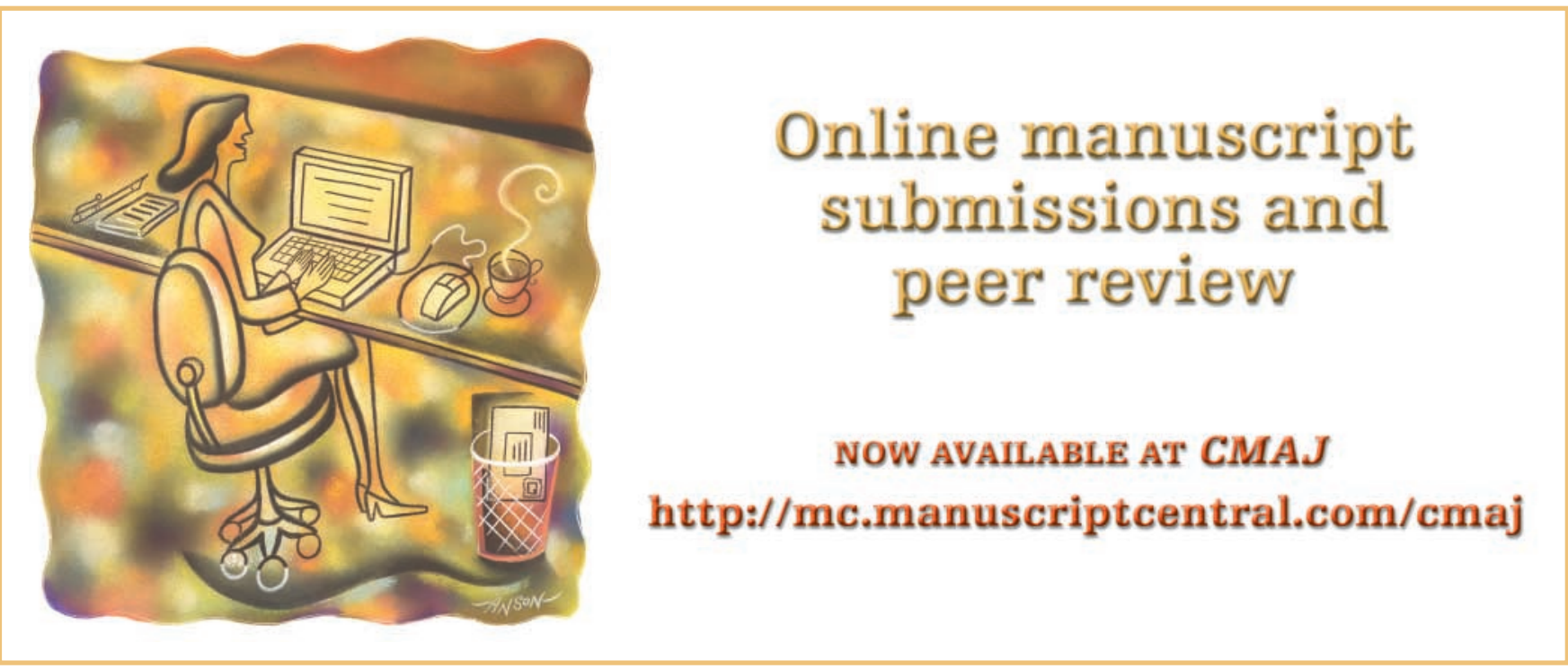

